\title{
Front Matter: Volume 8166
}

, "Front Matter: Volume 8166," Proc. SPIE 8166, Photomask Technology 2011, 816601 (3 November 2011); doi: 10.1117/12.917331

SDIE Event: SPIE Photomask Technology, 2011, Monterey, California, United SPIE. States 


\title{
Photomask Technology 2011
}

\author{
Wilhelm Maurer \\ Frank E. Abboud \\ Editors
}

19-22 September 2011

Monterey, California, United States

Sponsored by

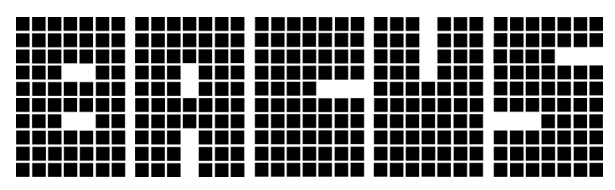

The international technical group of SPIE dedicated

to the advancement of photomask technology

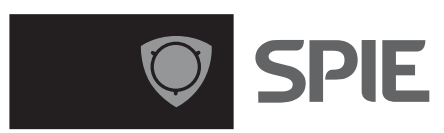

Published by

SPIE

Volume 8166

Proceedings of SPIE, 0277-786X, v. 8166 
The papers included in this volume were part of the technical conference cited on the cover and title page. Papers were selected and subject to review by the editors and conference program committee. Some conference presentations may not be available for publication. The papers published in these proceedings reflect the work and thoughts of the authors and are published herein as submitted. The publisher is not responsible for the validity of the information or for any outcomes resulting from reliance thereon.

Please use the following format to cite material from this book:

Author(s), "Title of Paper," in Photomask Technology 2011, edited by Wilhelm Maurer, Frank E. Abboud, Proceedings of SPIE Vol. 8166 (SPIE, Bellingham, WA, 2011) Article CID Number.

ISSN 0277-786X

ISBN 9780819487919

Published by

SPIE

P.O. Box 10, Bellingham, Washington 98227-0010 USA

Telephone +1 3606763290 (Pacific Time) · Fax +1 3606471445

SPIE.org

Copyright () 2011, Society of Photo-Optical Instrumentation Engineers

Copying of material in this book for internal or personal use, or for the internal or personal use of specific clients, beyond the fair use provisions granted by the U.S. Copyright Law is authorized by SPIE subject to payment of copying fees. The Transactional Reporting Service base fee for this volume is $\$ 18.00$ per article (or portion thereof), which should be paid directly to the Copyright Clearance Center (CCC), 222 Rosewood Drive, Danvers, MA 01923. Payment may also be made electronically through CCC Online at copyright.com. Other copying for republication, resale, advertising or promotion, or any form of systematic or multiple reproduction of any material in this book is prohibited except with permission in writing from the publisher. The CCC fee code is 0277-786X/11/ \$18.00.

Printed in the United States of America.

Publication of record for individual papers is online in the SPIE Digital Library.

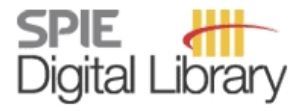

SPIEDigitalLibrary.org

Paper Numbering: Proceedings of SPIE follow an e-First publication model, with papers published first online and then in print and on CD-ROM. Papers are published as they are submitted and meet publication criteria. A unique, consistent, permanent citation identifier (CID) number is assigned to each article at the time of the first publication. Utilization of CIDs allows articles to be fully citable as soon as they are published online, and connects the same identifier to all online, print, and electronic versions of the publication. SPIE uses a six-digit CID article numbering system in which:

- The first four digits correspond to the SPIE volume number.

- The last two digits indicate publication order within the volume using a Base 36 numbering system employing both numerals and letters. These two-number sets start with 00, 01, 02, 03, 04 , $05,06,07,08,09,0 A, 0 B \ldots 0 Z$, followed by 10-1Z, 20-2Z, etc.

The CID number appears on each page of the manuscript. The complete citation is used on the first page, and an abbreviated version on subsequent pages. Numbers in the index correspond to the last two digits of the six-digit CID number. 


\title{
Contents
}

\section{Part One}

\author{
xvii Conference Committee \\ xxi Introduction \\ xxiii Schedule of Special Session on Is it too late to PANIC? EUV is real
}

INVITED SESSION

8166 OD Mask Industry Assessment: 2011 (Invited Paper) [8166-02]

Y. D. Chan, SEMATECH (United States)

8166 OE Additional evidence of EUV blank defects first seen by wafer printing [8166-03]

R. Jonckheere, D. Van den Heuvel, IMEC (Belgium); T. Bret, T. Hofmann, Carl Zeiss SMS GmbH (Germany); J. Magana, Intel Corp. (United States); I. Aharonson, D. Meshulach, AMAT PDC

(Israel); E. HendrickX, K. Ronse, IMEC (Belgium)

\section{EUV INFRASTRUCTURE AND APPLICATION I}

8166 OF Accelerating EUV learning with synchrotron light: mask roughness challenges ahead (Invited Paper) [8166-04]

P. P. Naulleau, K. A. Goldberg, E. Gullikson, I. Mochi, Lawrence Berkeley National Lab. (United States); B. McClinton, Univ, of California, Berkeley (United States); A. Rastegar, SEMATECH (United States)

$81660 G$ Phase defect analysis with actinic full-field EUVL mask blank inspection [8166-05] T. Yamane, T. Tanaka, T. Terasawa, O. Suga, MIRAl-Semiconductor Leading Edge Technologies, Inc. (Japan)

$8166 \mathrm{OH}$ Printability of native blank defects and programmed defects and their stack structures [8166-06]

H. J. Kwon, J. Harris-Jones, R. Teki, A. Cordes, SEMATECH (United States); T. Nakajima, AGC Electronics America (United States); I. Mochi, K. A. Goldberg, Lawrence Berkeley National Lab. (United States); Y. Yamaguchi, H. Kinoshita, Univ. of Hyogo (Japan)

8166 0J EUV mask absorber and multi-layer defect disposition techniques using computational lithography [8166-08]

V. Tolani, M. Satake, P. Hu, D. Peng, Y. Li, D. Kim, L. Pang, Luminescent Technologies, Inc. (United States) 
8166 OK Mask cycle time reduction for foundry projects [8166-09]

A. Balasinski, Cypress Semiconductor (United States)

SIMULATION OF MASK MAKING AND APPLICATION

8166 OM More than monitoring: advanced lithographic process tuning [8166-12]

G. R. Cantrell, J. A. Dumaya, C. Bürgel, A. Feicke, M. Häcker, C. Utzny, Advanced Mask Technology Ctr. GmbH \& Co. KG (Germany)

816600 Enabling virtual wafer CD (WCD) using inverse pattern rendering (IPR) of mask CD-SEM images [8166-87]

T. Dam, D. Chen, H.-M. Chang, N. Corcoran, P. Yu, L. Pang, Luminescent Technologies, Inc. (United States); C.-W. Chang, R. Lai, P. Chang, L. Tuo, Taiwan Semiconductor Manufacturing Co. Ltd. (Taiwan)

8166 OP PPF-Explorer: pointwise proximity function calibration using a new radial symmetric calibration structure [8166-88]

R. Galler, M. Krueger, D. Melzer, EQUIcon Software GmbH (Germany); L. E. Ramos, Vistec Electron Beam GmbH (Germany); M. Suelzle, EQUlcon Software GmbH (Germany);

U. Weidenmueller, Vistec Electron Beam GmbH (Germany)

\section{MASK DATA PREPARATION AND PROCESS CORRECTION}

$81660 Q$ OPC modeling and correction solutions for EUV lithography (Invited Paper) [8166-14] J. Word, C. Zuniga, M. Lam, Mentor Graphics Corp. (United States); M. Habib, Mentor Graphics Corp. (Egypt); K. Adam, M. Oliver, Mentor Graphics Corp. (United States)

8166 OR High speed mask inspection data prep flow based on pipelining [8166-15]

D. Hung, D. Morales, J. P. Canepa, S. Kim, Synopsys, Inc. (United States); P. Liu, J.-P. Sier, P. LoPresti, KLA-Tencor Corp. (United States)

8166 OS QOR analysis of fractured data solutions using distributed processing [8166-16]

B. D. S. S., N. Rao, R. R. Pai, N. P. Bhat, SoftJin Technologies Pvt. Ltd. (India)

8166 OT Reducing shot count through optimization-based fracture [8166-17]

T. Lin, E. Sahouria, N. Akkiraju, S. Schulze, Mentor Graphics Corp. (United States)

$81660 \mathrm{U}$ Application of signal reconstruction techniques to shot count reduction in simulation driven fracturing [8166-18]

S. Jiang, A. Zakhor, Univ. of California, Berkeley (United States) 
DESIGN FOR MANUFACTURABILITY, AND OPTICAL ENHANCEMENTS: SMO, OPC ETC. I

$8166 \mathrm{OV}$ SMO applied to contact layers at the $32 \mathrm{~nm}$ node and below with consideration of MEEF and MRC [8166-19]

T.-H. WU, SAMSUNG Electronics Co., Ltd. (Korea, Republic of); R. Sinn, B. Gleason, Luminescent Technologies, Inc. (United States); J. Kim, J. Hong, S. Park, S. Lee, SAMSUNG Electronics Co., Ltd. (Korea, Republic of)

8166 oW Evaluation of the accuracy of complex illuminator designs [8166-20] M. S. Hibbs, IBM Systems and Technology Group (United States); J. Tirapu-Azpiroz, IBM Microelectronics (United States); K. Seki, Toppan Photomasks, Inc. (United States); G. McIntyre, IBM Systems and Technology Group (United States); S. Kondo, Toppan Photomasks, Inc. (United States)

8166 0X Double patterning from design enablement to verification [8166-21]

D. Abercrombie, P. Lacour, Mentor Graphics Corp. (United States); O. El-Sewefy, Mentor Graphics Corp. (Egypt); A. Volkov, E. Levine, K. Arb, C. Reid, Q. Li, P. Ghosh, Mentor Graphics Corp. (United States)

8166 OY Study on design rule verification procedure of semiconductor memory devices by using design based metrology (DBM) [8166-22]

J. Jeong, S. Choi, S. Chang, M. Shim, G. Jin, SAMSUNG Electronics Co., Ltd. (Korea, Republic of)

\section{EUV INFRASTRUCTURE AND APPLICATION II}

$81660 Z$ Holistic lithography for EUV: NXE:3100 characterization of first printed wafers using an advanced scanner model and scatterometry (Invited Paper) [8166-23]

F. A. J. M. Driessen, N. Davydova, ASML Netherlands B.V. (Netherlands); J. Jiang, ASML Brion Technologies, Inc. (United States); H. Kang, ASML Technology Development Ctr. (United States); V. Vaenkatesan, D. Oorschot, ASML Netherlands B.V. (Netherlands); I. S. Kim, S. N. Kang, Y. Lee, J. Yeo, SAMSUNG Electronics Co., Ltd. (Korea, Republic of); K. Gronlund, H. Y. Liu, ASML Brion Technologies, Inc. (United States); K. van Ingen Schenau, R. Peeters, C. Wagner, ASML Netherlands B.V. (Netherlands); J. Zimmermann, O. Schumann, Carl Zeiss SMT GmbH (Germany)

816610 Development status and infrastructure progress update of aerial imaging measurements on EUV masks [8166-24]

S. Perlitz, W. Harnisch, U. Strößner, J. H. Peters, Carl Zeiss SMS GmbH (Germany); M. Weiss,

D. Hellweg, Carl Zeiss SMT GmbH (Germany)

816611 EUV mask preparation considering blank defects mitigation [8166-25]

Y. Du, H. Zhang, M. D. F. Wong, Univ. of Illinois at Urbana-Champaign (United States);

R. O. Topaloglu, GLOBALFOUNDRIES Inc. (United States)

\section{MASK CLEANING, CONTAMINATION, HAZE, AND PREVENTION I}

816613 A study on irregular growing defect mechanism and removal process [8166-27]

H. Lee, J. Jun, G. Jeong, S. Kim, S. Kim, C. Kim, Hynix Semiconductor Inc. (Korea, Republic of) 
816614 Megasonic cleaning: possible solutions for 22nm node and beyond [8166-29] H. Shende, MP Mask Technology Ctr., LLC (United States); S. Singh, SUSS MicroTec Inc. (United States); J. Baugh, R. Mann, MP Mask Technology Ctr., LLC (United States); U. Dietze, SUSS MicroTec Inc. (United States); P. Dress, HamaTech APE GmbH (Germany)

816615 Extending $\mathrm{CO}_{2}$ cryogenic aerosol cleaning for advanced optical and EUV mask cleaning [8166-30]

I. Varghese, C. W. Bowers, M. Balooch, Eco-Snow Systems, Rave N.P. Inc. (United States)

MASK PROCESSES, SUBSTRATES, AND MATERIALS I

816616 Advanced electron beam resist requirements and challenges (Invited Paper) [8166-31] A. Jamieson, Y. K. Kim, B. Olson, M. LU, N. Wilcox, Intel Corp. (United States)

816617 High resolution mask process and substrate for $20 \mathrm{~nm}$ and early $14 \mathrm{~nm}$ node lithography [8166-32]

T. Faure, IBM Systems and Technology Group (United States); S. Akutagawa, Toppan Photomasks, Inc. (United States); K. Badger, L. Kindt, IBM Systems and Technology Group (United States); J. Kotani, T. Mizoguchi, S. Nemoto, K. Seki, T. Senna, Toppan Photomasks, Inc. (United States); R. Wistrom, IBM Systems and Technology Group (United States); S. Igarashi, Y. Inazuki, K. Nishikawa, H. Yoshikawa, Shin-Etsu Chemical Co., Ltd. (Japan)

816618 Phase-shifting effect of thin-absorber EUV masks [8166-33]

H. Tanabe, T. Murachi, Intel K. K. (Japan); S. H. Lee, M. Chandhok, S.-J. Park, G. Zhang, Intel Corp. (United States); T. Abe, T. Ogase, N. Hayashi, Dai Nippon Printing Co., Ltd. (Japan)

816619 A study of closed-loop application: WLCD-CDC for 32nm and beyond reticles [8166-144] A. Goonesekera, Carl Zeiss NTS LLC (United States); U. Buttgereit, T. Thaler, Carl Zeiss SMS GmbH (Germany); E. Graitzer, Carl Zeiss SMS Ltd. (Israel)

\section{MASK PATTERN GENERATION I}

8166 1B The requirements for the future e-beam mask writer: statistical analysis of pattern accuracy [8166-36]

S. H. Lee, J. Choi, H. B. Kim, B. G. Kim, H.-K. Cho, SAMSUNG Electronics Co., Ltd. (Korea, Republic of)

$81661 \mathrm{C}$ The trouble starts with using electrons: putting charging effect correction models to the test [8166-37]

T. Wandel, C. Utzny, Advanced Mask Technology Ctr. Dresden (Germany); N. Nakayamada, NuFlare Technology, Inc. (Japan)

8166 ID EBM-8000: EB mask writer for product mask fabrication of $22 \mathrm{~nm}$ half-pitch generation and beyond [8166-38]

S. Yoshitake, T. Kamikubo, N. Nakayamada, K. Hattori, H. Ando, T. lijima, K. Ohtoshi, K. Saito, R. Yoshikawa, S. Tamamushi, R. Tomiyoshi, H. Higurashi, Y. Hattori, S. Tsuchiya, M. Katoh,

K. Suzuki, Y. Tachikawa, M. Ogasawara, NuFlare Technology, Inc. (Japan); V. Katsap,

S. Golladay, R. Kendall, NuFlare Technology, Inc. (United States) 
$81661 \mathrm{E}$ In-die photomask registration and overlay metrology with PROVE using 2D correlation methods [8166-39]

D. Seidel, Carl Zeiss SMS GmbH (Germany); M. Arnz, Carl Zeiss SMT GmbH (Germany);

D. Beyer, Carl Zeiss SMS GmbH (Germany)

8166 IF Evaluation of KLA-Tencor LMS IPRO5 beta system for $22 \mathrm{~nm}$ node registration and overlay applications [8166-40]

M. Ferber, F. Laske, K.-D. Röth, D. Adam, KLA-Tencor MIE GmbH (Germany)

\section{MASK INSPECTION AND REPAIR I}

$81661 G \quad$ Repair of natural EUV reticle defects [8166-42]

R. Jonckheere, IMEC (Belgium); T. Bret, Carl Zeiss SMS GmbH (Germany); D. Van den Heuvel, IMEC (Belgium); J. Magana, Intel Corp. (United States); W. Gao, Synopsys Inc. (Belgium); M. Waiblinger, Carl Zeiss SMS GmbH (Germany)

$81661 \mathrm{H} \quad$ EUVL mask inspection using 193nm wavelength for 30nm node and beyond [8166-43] J. Na, W. Cho, T.-G. Kim, I.-Y. Kang, B. Cha, I. Shin, H.-K. Cho, SAMSUNG Electronics Co., Ltd. (Korea, Republic of)

$816611 \quad$ Efficiency and throughput improvement on defect disposition through automated defect classification [8166-100]

L. He, N. Corcoran, D. Peng, V. Tolani, H.-M. Chang, P. Yu, K. Wang, Luminescent Technologies, Inc. (United States); C. J. Chen, T. H. Yen, R. Lai, B. H. Ong, L. C. C. Tuo, Taiwan Semiconductor Manufacturing Co. Ltd. (Taiwan)

$81661 \mathrm{~J} \quad$ Clean and repair of EUV photomasks [8166-45]

T. Robinson, D. Yi, D. Brinkley, K. Roessler, R. White, R. Bozak, M. Archuletta, D. Lee, RAVE LLC (United States)

\section{MASK CLEANING, CONTAMINATION, HAZE, AND PREVENTION II}

$81661 \mathrm{~K}$ Challenges associated with advanced mask cleaning (Invited Paper) [8166-46]

B. J. Grenon, Grenon Consulting, Inc. (United States)

$81661 \mathrm{~L}$ Study on the soft defects related to dry etch process of phase shift mask [8166-47] Y.-J. An, J.-M. Kim, B.-S. Kang, D.-H. Lee, S.-S. Choi, PKL Co., Ltd. (Korea, Republic of)

$81661 \mathrm{M}$ Effect of SPM-based cleaning POR on EUV mask performance [8166-48]

J. Choi, SAMSUNG Electronics Co., Ltd. (Korea, Republic of) and SEMATECH (United States); H. Lee, J. Yoon, SAMSUNG Electronics Co., Ltd. (Korea, Republic of); T. Shimomura, DNP USA (United States); A. Friz, IBM (United States) and SEMATECH (United States); C. Montgomery, SEMATECH (United States); A. Ma, Intel Corp. (United States) and SEMATECH (United States); F. Goodwin, SEMATECH (United States); D. Kang, P. Chung, I. Shin, H. Cho, SAMSUNG Electronics Co., Ltd. (Korea, Republic of) 
8166 iN Scanning probe microscopy study of the tolerance of patterned EUV masks to megasonic cleaning [8166-49]

T. Shimomura, DNP USA (United States); A. Rastegar, SEMATECH (United States)

816610 Effect of repetitive acid-based cleaning on EUV mask lifetime and lithographic performance [8166-50]

R. J. Chen, Intel Corp. (United States); B. M. McClinton, S. A. George, Lawrence Berkeley

National Lab. (United States); Y. Kim, Intel Corp. (United States); L.-M. Baclea-an,

P. P. Naulleau, Lawrence Berkeley National Lab. (United States)

\section{MASK METROLOGY II}

8166 IP EUV and $x$-ray scattering methods for CD and roughness measurement [8166-52] F. Scholze, A. Kato, J. Wernecke, M. Krumrey, Physikalisch-Technische Bundesanstalt (Germany)

8166 IQ Investigation of 3D patterns on EUV masks by means of scatterometry and comparison to numerical simulations [8166-53]

S. Burger, Zuse Institute Berlin (Germany) and JCMwave GmbH (Germany); L. Zschiedrich, J. Pomplun, JCMwave GmbH (Germany); F. Schmidt, Zuse Institute Berlin (Germany) and JCMwave GmbH (Germany); A. Kato, C. Laubis, F. Scholze, Physikalisch-Technische Bundesanstalt (Germany)

8166 IR Addressing 3D metrology challenges by using a multiple detector CDSEM [8166-54] M. Hiroyama, T. Murakawa, M. Kuribara, T. Iwai, M. Soma, I. Iko, M. Seyama, J. Matsumoto, T. Nakamura, Advantest Corp. (Japan); H. Hakii, I. Yonekura, M. Kawashita, Y. Nishiyama, K. Tanaka, K. Komoto, Toppan Printing Co., Ltd. (Japan)

8166 is The assessment of the impact of mask pattern shape variation on the OPC-modeling by using SEM-contours from wafer and mask [8166-55]

D. Hibino, H. Shindo, Y. Hojyo, Hitachi High-Technologies Corp. (Japan); T. Do, A. Dave, T. Lin, I. Kusnadi, J. L. Sturtevant, Mentor Graphics Corp. (United States)

\section{NIL INFRASTRUCTURE AND APPLICATION I}

8166 IU 30nm full field quartz template replicated from Si master for FLASH active layer NIL [8166-58] D. Lee, B.-K. Lee, W. Ko, J.-K. Kim, K. Yang, SAMSUNG Advanced Institute of Technology (Korea, Republic of); B. Seung, I. Jang, M. J. Kim, B. Kim, C. Park, J. Yeo, SAMSUNG Electronics Co., Ltd. (Korea, Republic of); C. Moon, SAMSUNG Advanced Institute of Technology (Korea, Republic of)

$81661 \mathrm{~V}$ Fabrication of $20 \mathrm{~nm}$ half-pitch quartz template by nano-imprinting [8166-120] N. Sato, T. Oomatsu, S. Wakamatsu, K. Nishimaki, T. Usa, K. Kodama, K. Usuki, FU JIFILM Corp. (Japan) 
8166 IW Dry etching performance of advanced EUV mask blanks [8166-59] J. Whang, IBM Microelectronics (United States); M. Chandrachood, Applied Materials, Inc. (United States); E. Gallagher, T. Faure, IBM Microelectronics (United States); M. Grimbergen, Applied Materials, Inc. (United States); S. Crawford, IBM Microelectronics (United States); K. YU, T. Y. B. Leung, Applied Materials, Inc. (United States); R. Wistrom, IBM Microelectronics (United States); A. Sabharwal, J. Chen, B. Wu, Applied Materials, Inc. (United States)

$81661 \mathrm{Y}$ The impact of a thinner binary mask absorber on $22 \mathrm{~nm}$ and beyond mask inspectability and defect sensitivity [8166-61]

K. D. Badger, IBM Systems and Technology Group (United States); K. Seki, Toppan

Photomasks, Inc. (United States)

\section{Part Two}

\section{MASK PATTERN GENERATION II}

816612 Pattern placement error due to resist charging effect at 50kV e-beam writer: mechanism and its correction [8166-62]

J. Choi, S. J. Bae, H. B. Kim, B. G. Kim, H. K. Cho, SAMSUNG Electronics Co., Ltd. (Korea, Republic of)

816620 Enhancement of global CD correction and data processing in EB mask writer EBM-8000 [8166-63]

H. Matsumoto, Y. Kato, T. Motosugi, J. Yashima, T. Abe, N. Nakayamada, S. Yoshitake, K. Hattori, NuFlare Technology, Inc. (Japan)

\section{NEW MASK MAKING AND ALTERNATIVES I}

816621 Efficient large volume data preparation for electron beam lithography for sub-45nm node [8166-64]

K.-H. Choi, M. Gutsch, M. Freitag, C. Hohle, Fraunhofer Ctr. Nanoelectronic Technologies (Germany); L. Martin, S. Bayle, S. Manakli, P. Schiavone, Aselta Nanographics, MINATEC-BHT (France)

816622 eMET POC: realization of a proof-of-concept $50 \mathrm{keV}$ electron multibeam mask exposure tool [8166-65]

E. Platzgummer, C. Klein, H. Loeschner, IMS Nanofabrication AG (Austria)

816623 CD error budget of CP exposure [8166-66]

M. Kurokawa, H. Isobe, M. Takizawa, K. Bunya, H. Komami, A. Yamada, Advantest Corp. (Japan); K. Shiomitsu, K. Oonou, Toppan Printing Co., Ltd. (Japan) 
816624 Mask aspects of EUVL imaging at 27nm node and below [8166-67]

N. Davydova, E. van Setten, ASML Netherlands B.V. (Netherlands); S.-I. Han, ASML Albany (United States); M. van de Kerkhof, R. de Kruif, D. Oorschot, ASML Netherlands B.V.

(Netherlands); J. Zimmerman, ASML Wilton (United States); A. Lammers, ASML Netherlands B.V. (Netherlands); B. Connolly, Toppan Photomasks Inc (Germany); F. Driessen,

A. van Oosten, ASML Netherlands B.V. (Netherlands); M. Dusa, ASML Belgium (Belgium);

Y. van Dommelen, ASML Albany (United States); N. Harned, ASML Wilton (United States);

J. Jiang, W. Liu, H. Kang, H. Liu, Brion Technologies, Inc. (United States)

\section{NIL INFRASTRUCTURE AND APPLICATION II}

816626 Imprint lithography template technology for bit patterned media (BPM) [8166-69]

J. Lille, K. Patel, R. Ruiz, T.-W. Wu, H. Gao, L. Wan, G. Zeltzer, E. Dobisz, T. R. Albrecht, Hitachi San Jose Research Ctr. (United States)

816627 Mask replication using jet and flash imprint lithography [8166-70]

K. S. Selinidis, C. Jones, G. F. Doyle, L. Brown, J. Imhof, D. L. LaBrake, D. J. Resnick, S. V. Sreenivasan, Molecular Imprints, Inc. (United States)

DESIGN FOR MANUFACTURABILITY, AND OPTICAL ENHANCEMENTS: SMO, OPC ETC. II

816629 Exploring the impact of mask making constraints on double patterning design rules [8166-72]

T. Dam, R. Sinn, P. Rissman, B. Gleason, Luminescent Technologies, Inc. (United States)

8166 2A A new source optimization approach for 2X node logic [8166-74]

K. Iwase, Sony Corp. (Belgium); P. De Bisschop, IMEC (Belgium); B. Laenens, Z. Li, K. Gronlund, ASML Brion Technologies, Inc. (United States); P. van Adrichem, ASML Brion Technologies, Inc. (United States) and ASML (Netherlands); S. HsU, ASML Brion Technologies, Inc. (United States)

\section{NEW MASK MAKING AND ALTERNATIVES II}

8166 2B Si stencil masks for organic thin film transistor fabrication (Invited Paper) [8166-75]

F. Letzkus, T. Zaki, IMS Chips (Germany); F. Ante, Max Planck Institute for Solid State Research (Germany); H. Richter, J. N. Burghartz, J. Butschke, IMS Chips (Germany); H. Klauck, Max Planck Institute for Solid State Research (Germany)

8166 2C Bottlenecks in data preparation flow for multi-beam direct write [8166-76]

D. Hung, Synopsys, Inc. (United States); O. Meijer, Synopsys Netherlands B.V. (United States); A. Zepka, Synopsys, Inc. (United States)

\section{MASK INSPECTION AND REPAIR II}

8166 2D Simulation based mask defect printability verification and disposition. Part II [8166-82] E. Guo, I. Shi, B. Gao, N. Fan, Semiconductor Manufacturing International Corp. (China); G. Cheng, L. Ling, K. Zhou, G. Zhang, Anchor Semiconductor (China); Y. Chen, C. Hsiang, B. Su, Anchor Semiconductor (United States) 
$81662 \mathrm{E}$ EUV multilayer defect compensation (MDC) by absorber pattern modification: from theory to wafer validation [8166-83]

L. Pang, P. Hu, M. Satake, V. Tolani, D. Peng, Y. Li, D. Chen, Luminescent Technologies, Inc. (United States)

$81662 \mathrm{~F} \quad$ Performance of EBeyeM for EUV mask inspection [8166-84]

S. Yamaguchi, M. Naka, T. Hirano, M. Itoh, M. Kadowaki, T. Koike, Y. Yamazaki, Toshiba Corp. (Japan); K. Terao, M. Hatakeyama, K. Watanabe, H. Sobukawa, T. Murakami, T. Karimata,

K. Tsukamoto, T. Hayashi, R. Tajima, N. Kimura, EBARA Corp. (Japan); N. Hayashi, Dai Nippon Printing Co., Ltd. (Japan)

SPECIAL SESSION: IS IT TOO LATE TO PANIC? EUV IS REAL!

816621 Challenges for 1x-nm device fabrication using EUVL: scanner and mask (Invited Paper) [8166-77]

W. H. Arnold, ASML Technology Development Ctr. (United States)

POSTER SESSION: MASK BUSINESS

$81662 \mathrm{~N}$ Reticle process monitoring and qualification based on reticle CDU and wafer CDU correlation [8166-89]

G. Ning, GLOBALFOUNDRIES Dresden Module One LLC \& Co. KG (Germany); B. I. Choi, GLOBALFOUNDRIES Singapore Pte. Ltd. (Singapore); C. Holfeld, GLOBALFOUNDRIES Dresden Module One LLC \& Co. KG (Germany); Y. T. Ngow, S. K. Tan, GLOBALFOUNDRIES Singapore Pte. Ltd. (Singapore); A. Tchikoulaeva, GLOBALFOUNDRIES Dresden Module Two LLC \& Co. KG (Germany); F. H. Gn, GLOBALFOUNDRIES Singapore Pte. Ltd. (Singapore)

POSTER SESSION: MASK CLEANING, CONTAMINATION, HAZE, AND PREVENTION

$81662 \mathrm{P}$ Effects of cleaning on NIL templates: surface roughness, CD, and pattern integrity [8166-91] S. Singh, SUSS MicroTec Inc. (United States); K. Onuki, SUSS MicroTec KK (Japan); N. Kawamata, T. Nagai, M. Kurihara, T. Tomita, N. Hayashi, Dai Nippon Printing Co., Ltd. (Japan); P. Dress, HamaTech APE GmbH (Germany); U. Dietze, SUSS MicroTec Inc. (United States)

$81662 Q \quad$ Study on EUV photomask resist stripping and cleaning [8166-93] T. Kikuchi, Y. Nagashima, H. Nakano, T. Wakatsuki, K. Demura, Y. Kurokawa, M. Nonaka, Shibaura Mechatronics Corp. (Japan)

POSTER SESSION: EUV INFRASTRUCTURE AND APPLICATION

816625 Investigating the intrinsic cleanliness of automated handling designed for EUV mask pod-in-pod systems [8166-95]

O. Brux, HamaTech APE GmbH \& Co. KG (Germany); P. van der Walle, J. C. J. van der

Donck, TNO (Netherlands); P. Dress, HamaTech APE GmbH \& Co. KG (Germany) 
$81662 T$ Optimized qualification protocol on particle cleanliness for EUV mask infrastructure [8166-96]

J. C. J. van der Donck, J. K. Stortelder, G. B. Derksen, TNO (Netherlands)

$81662 U$ EQ-10 electrodeless Z-pinch EUV source for metrology applications [8166-97]

D. Gustafson, S. F. Horne, M. J. Partlow, M. M. Besen, D. K. Smith, P. A. Blackborow, Energetiq Technology, Inc. (United States)

POSTER SESSION: MASK INSPECTION AND REPAIR

$81662 \mathrm{~V}$ Improve the efficiency of the inspection process via a thorough control of the scanning focus [8166-98]

E. Villa, L. Sartelli, H. Miyashita, DNP Photomask Europe (Italy)

$81662 \mathrm{~W} \quad$ Electron beam EUV patterned mask inspection system [8166-101]

K. Yamada, Y. Kitayama, Holon Corp. (Japan); P. Fiekowsky, Automated Visual Inspection LLC (United States)

$81662 \mathrm{X}$ Productivity of femtosecond DUV laser photomask repair in a real world mask house [8166-103]

J.-H. Lin, C. Y. Chen, F. G. Tsai, Taiwan Semiconductor Manufacturing Co. Ltd. (Taiwan);

T. Robinson, D. Yi, J. LeClaire, R. White, R. Bozak, M. Archuletta, RAVE LLC (United States)

$81662 Y$ Nanomachining repair for the latest reticle enhancement technologies [8166-104]

T. Robinson, D. Yi, D. Brinkley, K. Roessler, R. White, R. Bozak, M. Archuletta, B. Arruza, RAVE

LLC (United States)

816622 Parallelized automatic false defect detection using GPUs during mask inspection [8166-105] M. Pereira, M. Maji, B. Gangadhar, R. R. Pai, I. Nigam, A. Parchuri, Soft Jin Technologies Pvt. Ltd. (India)

\section{POSTER SESSION: MASK DATA PREPARATION AND PROCESS CORRECTION}

816632 Optimization of mask shot count using MB-MDP and lithography simulation (First Place, Best Poster Award) [8166-110]

G. S. Chua, W. L. Wang, B. I. Choi, GLOBALFOUNDRIES Singapore (Singapore); Y. Zou,

C. Tabery, GLOBALFOUNDRIES Inc. (United States); I. Bork, T. Nguyen, A. Fujimura, D2S, Inc. (United States)

816634 Assessment and comparison of different approaches for mask write time reduction [8166-151]

A. Elayat, T. Lin, E. Sahouria, S. F. Schulze, Mentor Graphics Corp. (United States)

\section{POSTER SESSION: MASK METROLOGY}

816639 In-die mask registration measurement with existing inspection tools [8166-118]

S. Tamamushi, T. Touya, NuFlare Technology, Inc. (Japan) 
$81663 \mathrm{~A}$ The influence and improvement of through pellicle image placement [8166-119] W. C. Lo, Y. F. Cheng, M. J. Chen, United Microelectronics Corp. (Taiwan); K. Takushima, S. Tashiro, HOYA Corp. (Japan)

\section{POSTER SESSION: NIL INFRASTRUCTURE AND APPLICATION}

$81663 \mathrm{~B}$ Releasing material screening and continuous nano-imprinting in mold replication for nano-imprint lithography [8166-121]

K. Suzuki, H. Kobayashi, T. Sato, H. Yamashita, T. Watanabe, HOYA Corp. (Japan)

POSTER SESSION: DESIGN FOR MANUFACTURABILITY, AND OPTICAL ENHANCEMENTS: SMO, RET, OPC, ETC.

$81663 \mathrm{C}$ Freeform source optimization for improving litho-performance of warm spots [8166-73] C.-W. Wu, C.-C. Liao, C.-L. Shih, Nanya Technology Corp. (Taiwan); C.-H. Chang, S. Hsu, H.-Y. Liu, Z. Li, ASML Brion Technologies, Inc. (United States)

$81663 \mathrm{D}$ Layout decomposition and mask synthesis for double and triple exposure with image reversal in a single photoresist layer (Third Place, Best Poster Award) [8166-122] C. Ndoye, M. Orlowski, Virginia Tech (United States)

$81663 \mathrm{E} \quad$ Hot spot detection for indecomposable self-aligned double patterning layout [8166-123] H. Zhang, Y. Du, M. D. F. Wong, Univ. of Illinois at Urbana-Champaign (United States); R. O. Topaloglu, GLOBALFOUNDRIES Inc. (United States)

$81663 \mathrm{G}$ Determination of CD variance factors of $28 \mathrm{~nm} 1 \mathrm{x}$-metal layer hotspots using experimental and simulated CD contours [8166-125]

F. Weisbuch, J. Schramm, GLOBALFOUNDRIES Dresden (Germany)

$81663 \mathrm{H} \quad$ Yield optimization through MLR techniques [8166-126]

P. Morey-Chaisemartin, E. Beisser, XYALIS (France)

816631 Dynamic feedback controller for optical proximity correction [8166-128] A. Omran, Mentor Graphics Corp. (Egypt); J. Schact, J. Pan, Mentor Graphics Corp. (Taiwan); J. Lei, L. Hong, Mentor Graphics Corp. (United States); M. Al-Imam, Mentor Graphics Corp. (Egypt); N. Cobb, Mentor Graphics Corp. (United States); R. Shen, R. Chou, Mentor Graphics Corp. (Taiwan)

$81663 \mathrm{~J}$ Anticipation of dimensional issues caused by topography during photo lithography [8166-129]

L. Ravel, STMicroelectronics (France); C. Brault, Dow Advanced Materials (France); C. Hegaret, LFoundry (France); A. Di Giacomo, R. Lallement, J. Azémar, STMicroelectronics (France); M. Hellion, Dow Advanced Materials (France)

$81663 \mathrm{M}$ Total source mask optimization: high-capacity, resist modeling, and production-ready mask solution [8166-132] M. Fakhry, IBM Almaden Research Ctr. (United States); Y. Granik, K. Adam, Mentor Graphics Corp. (United States); K. Lai, IBM Semiconductor Research and Development Ctr. (United States) 
816630 Performance and variability driven guidelines for BEOL layout decomposition with LELE double patterning [8166-134]

T.-B. Chan, K. Jeong, A. B. Kahng, Univ. of California, San Diego (United States)

$81663 \mathrm{P}$ Integrated advanced hotspot analysis techniques in the post-OPC verification flow [8166-135]

M. Miyagi, P. Brooker, C. Sawh, T. Brist, K. Taravade, Synopsys, Inc. (United States)

$81663 Q \quad$ Double patterning for 56nm pitch test designs using inverse lithography [8166-136]

T. Dam, R. Gleason, P. Rissman, R. Sinn, Luminescent Technologies, Inc. (United States)

$81663 R \quad$ Using custom features to check OPC model performance [8166-137]

A. Abdo, R. Viswanathan, IBM Systems and Technology Group (United States)

POSTER SESSION: MASK PROCESSES, SUBSTRATES, AND MATERIALS

8166 3T Development status of EUVL mask blank and substrate [8166-140]

Y. Hirabayashi, Asahi Glass Co., Ltd. (Japan)

$81663 \mathrm{U}$ The trade-offs between thin and thick absorbers for EUV photomasks [8166-143]

G. McIntyre, IBM Advanced Lithography Research (United States); C. Zuniga, Mentor Graphics (United States); E. Gallagher, J. Whang, L. Kindt, IBM Photomask Development (United States)

8166 3V Aftenuated phase-shift mask with high tolerance for 193nm radiation damage (Second Place, Best Poster Award) [8166-142]

T. Yamazaki, R. Gorai, Y. Kojima, T. Haraguchi, T. Tanaka, Toppan Printing Co., Ltd. (Japan);

R. Koitabashi, Y. Inazuki, H. Yoshikawa, Shin-Etsu Chemical Co., Ltd. (Japan)

$81663 \mathrm{~W}$ Dry etching technologies for the advanced binary film [8166-145]

Y. lino, M. Karyu, H. Ita, T. Yoshimori, H. Azumano, M. Muto, M. Nonaka, Shibaura Mechatronics Corp. (Japan)

POSTER SESSION: SIMULATION OF MASK MAKING AND APPLICATIONS

$81663 Y$ Towards the prediction of pattern collapse hotspots for full-chip layouts [8166-147] J. L. Sturtevant, A. Dave, U. Hollerbach, Mentor Graphics Corp. (United States)

816632 Rigorous EMF simulation of the impact of photomask line-edge and line-width roughness on lithographic processes [8166-148]

O. H. Rudolph, P. Evanschitzky, A. Erdmann, E. Bär, J. Lorenz, Fraunhofer IISB (Germany)

816640 Accurate prediction of 3D mask topography induced best focus variation in full-chip photolithography applications [8166-149]

P. Liu, Brion Technologies, Inc. (United States) 
POSTER SESSION: NEW MASK MAKING AND ALTERNATIVES

816641 Improving the accuracy of the bimetallic grayscale photomasks using a feedback controlled flat-top beam [8166-150]

R. Qarehbaghi, G. H. Chapman, W. Boonyasiriwat, Simon Fraser Univ. (Canada)

POSTER SESSION: PHOTOMASK JAPAN 2011

8166 4D Printability and detection of backside defects on photomasks [8166-44]

G. Ning, C. Holfeld, GLOBALFOUNDRIES Dresden Module One LLC \& Co KG (Germany);

A. Tchikoulaeva, GLOBALFOUNDRIES Dresden Module Two, GmbH \& Co. KG (Germany); M. Sczyrba, Advanced Mask Technology Ctr. GmbH \& Co. KG (Germany); A. Ho, GLOBALFOUNDRIES Singapore Pte. Ltd. (Singapore); K. Bubke, GLOBALFOUNDRIES Dresden Module One LLC \& Co KG (Germany); S. Y. Tan, GLOBALFOUNDRIES Singapore Pte. Ltd. (Singapore); A. Holfeld, GLOBALFOUNDRIES Dresden Module One LLC \& Co. KG (Germany); B. I. Choi, GLOBALFOUNDRIES Singapore Pte. Ltd. (Singapore)

Author Index 
Downloaded From: https://www.spiedigitallibrary.org/conference-proceedings-of-spie on 25 Apr 2023

Terms of Use: https://www.spiedigitallibrary.org/terms-of-use 


\title{
Conference Committee
}

\author{
Conference Chair
}

Wilhelm Maurer, Infineon Technologies AG (Germany)

Conference Cochair

Frank E. Abboud, Intel Corporation (United States)

Program Committee

Arthur P. Balasinski, Cypress Semiconductor Corporation (United States)

Ronald R. Bozak, RAVE LLC (United States)

William H. Broadbent, KLA-Tencor Corporation (United States)

Peter D. Buck, Toppan Photomasks, Inc. (United States)

Emily E. Gallagher, IBM Corporation (United States)

Brian J. Grenon, Grenon Consulting, Inc. (United States)

Naoya Hayashi, Dai Nippon Printing Company, Ltd. (Japan)

Mark T. Jee, HOYA Corporation USA (United States)

Rik M. Jonckheere, IMEC (Belgium)

Bryan S. Kasprowicz, Photronics, Inc. (United States)

Byung-Gook Kim, SAMSUNG Electronics Company, Ltd. (Korea, Republic of)

Shy-Jay Lin, Taiwan Semiconductor Manufacturing Company Ltd.

(Taiwan)

M. Warren Montgomery, College of Nanoscale Science \& Engineering (United States)

Thomas H. Newman, Micronic Laser Systems Inc. (United States)

Hiroshi Nozue, NuFlare Technology, Inc. (Japan)

James E. Potzick, National Institute of Standards and Technology (United States)

Emmanuel Rausa, Plasma-Therm LLC (United States)

Douglas J. Resnick, Molecular Imprints, Inc. (United States)

Thomas Scherübl, Carl Zeiss SMS GmbH (Germany)

Steffen F. Schulze, Mentor Graphics Corporation (United States)

Robert J. Socha, ASML US, Inc. (United States)

Anna Tchikoulaeva, GLOBALFOUNDRIES Dresden Module Two, GmbH

\& Company KG (Germany)

Banqiu Wu, Applied Materials, Inc. (United States)

Larry S. Zurbrick, Agilent Technologies, Inc. (United States) 
Session Chairs

Photomask Japan Opening Remarks

Hiroaki Morimoto, Toppan Printing Company, Ltd. (Japan)

Naoya Hayashi, Dai Nippon Printing Company, Ltd. (Japan)

Photomask Japan Special Session

Hiroaki Morimoto, Toppan Printing Company, Ltd. (Japan)

Naoya Hayashi, Dai Nippon Printing Company, Ltd. (Japan)

Opening Remarks

Wilhelm Maurer, Infineon Technologies AG (Germany)

Frank E. Abboud, Intel Corporation (United States)

Keynote Session

Wilhelm Maurer, Infineon Technologies AG (Germany)

Frank E. Abboud, Intel Corporation (United States)

Invited Session

Frank E. Abboud, Intel Corporation (United States)

Wilhelm Maurer, Infineon Technologies AG (Germany)

EUV Infrastructure and Application I

Rik M. Jonckheere, IMEC (Belgium)

M. Warren Montgomery, College of Nanoscale Science \& Engineering (United States)

Mask Business

Naoya Hayashi, Dai Nippon Printing Company, Ltd. (Japan)

Bryan S. Kasprowicz, Photronics, Inc. (United States)

Simulation of Mask Making and Application

Larry S. Zurbrick, Agilent Technologies, Inc. (United States)

Peter D. Buck, Toppan Photomasks, Inc. (United States)

Mask Data Preparation and Process Correction

Peter D. Buck, Toppan Photomasks, Inc. (United States)

Wilhelm Maurer, Infineon Technologies AG (Germany)

Design for Manufacturability, and Optical Enhancements: SMO, OPC, etc. I

Byung-Gook Kim, SAMSUNG Electronics Company, Ltd. (Korea, Republic of)

Steffen F. Schulze, Mentor Graphics Corporation (United States) 
EUV Infrastructure and Application II

M. Warren Montgomery, College of Nanoscale Science \& Engineering (United States)

Rik M. Jonckheere, IMEC (Belgium)

Mask Cleaning, Contamination, Haze, and Prevention I

Brian J. Grenon, Grenon Consulting, Inc. (United States)

Anna Tchikoulaeva, GLOBALFOUNDRIES Dresden Module Two, GmbH \& Company KG (Germany)

Mask Processes, Substrates, and Materials I

Emmanuel Rausa, Plasma-Therm LLC (United States)

Banqiu Wu, Applied Materials, Inc. (United States)

Mask Pattern Generation I

Thomas H. Newman, Micronic Laser Systems Inc. (United States)

Shy-Jay Lin, Taiwan Semiconductor Manufacturing Company Ltd.

(Taiwan)

Mask Metrology I

Thomas Scherübl, Carl Zeiss SMS GmbH (Germany)

Larry S. Zurbrick, Agilent Technologies, Inc. (United States)

Mask Inspection and Repair I

Emily E. Gallagher, IBM Corporation (United States)

Ronald R. Bozak, RAVE LLC (United States)

Mask Cleaning, Contamination, Haze, and Prevention II

Anna Tchikoulaeva, GLOBALFOUNDRIES Dresden Module Two, GmbH

\& Company KG (Germany)

Brian J. Grenon, Grenon Consulting, Inc. (United States)

Mask Metrology II

James E. Potzick, National Institute of Standards and Technology (United States)

Emily E. Gallagher, IBM Corporation (United States)

NIL Infrastructure and Application I

Naoya Hayashi, Dai Nippon Printing Company, Ltd. (Japan)

Douglas J. Resnick, Molecular Imprints, Inc. (United States)

Mask Processes, Substrates, and Materials II

Mark T. Jee, HOYA Corporation USA (United States)

Bryan S. Kasprowicz, Photronics, Inc. (United States) 
Mask Pattern Generation II

Shy-Jay Lin, Taiwan Semiconductor Manufacturing Company Ltd.

(Taiwan)

Thomas H. Newman, Micronic Laser Systems Inc. (United States)

New Mask Making and Alternatives I

Artur P. Balasinski, Cypress Semiconductor Corporation (United States)

NIL Infrastructure and Application II

Douglas J. Resnick, Molecular Imprints, Inc. (United States)

Naoya Hayashi, Dai Nippon Printing Company, Ltd. (Japan)

Design for Manufacturability, and Optical Enhancements: SMO, OPC, etc. II

Steffen F. Schulze, Mentor Graphics Corporation (United States)

Byung-Gook Kim, SAMSUNG Electronics Company, Ltd. (Korea,

Republic of)

New Mask Making and Alternatives II

Artur P. Balasinski, Cypress Semiconductor Corporation (United States)

Mask Inspection and Repair II

Bryan S. Kasprowicz, Photronics, Inc. (United States)

John M. Whittey, KLA-Tencor Corporation (United States)

Special Session Opening Remarks

Frank E. Abboud, Intel Corporation (United States)

Robert J. Socha, ASML US, Inc. (United States)

Special Session: Is it too late to panic? EUV is Real!

Frank E. Abboud, Intel Corporation (United States)

Robert J. Socha, ASML US, Inc. (United States)

Best Paper and Poster Awards, and Prize Drawing

Wilhelm Maurer, Infineon Technologies AG (Germany)

Frank E. Abboud, Intel Corporation (United States)

International Poster Chairs

John M. Whittey, KLA-Tencor Corporation (United States)

Shy-Jay Lin, Taiwan Semiconductor Manufacturing Company Ltd.

(Taiwan)

Uwe Behringer, UBC Microelectronics (Germany) 


\section{Introduction}

This proceedings volume contains accepted papers from the SPIE conference on Photomask Technology 2011. The conference was arranged through the Bay Area Chrome Users Society (BACUS) and held as part of the 31st International Symposium on Photomask Technology, 19-22 September 2011 in Monterey, California, United States.

This year's conference introduced, as a new feature, a total of six invited papers in addition to the annual invited Mask Industry Assessment presentation by SEMATECH, and the best paper by EMLC 2011. These invited papers started corresponding key sessions with the insight of a competent, savvy expert in the corresponding field. During these invited presentations no parallel presentations were given, so every participant had a real chance to get a comprehensive overview of the key subjects in each field of Photomask.

The primary technical subject of this year's conference has been the arrival of EUV in the mainstream of mask technology. To affirm this statement, the program presented only topics very specific to EUV in two dedicated EUV sessions. All "regular" mask-related topics of EUV, e.g.: mask substrates, mask inspection, etc.; and results reported on EUV mask topics, in particular on mask defects, on their printability, potential prevention, and options to repair, were the basis of lively discussions, which could be overheard in many instances outside of the conference rooms. Everybody seriously involved in mask topics cannot get around EUV masks anymore, but has to find her/his potential contribution there.

The exact same statement was provided by Thursday's special session titled "Is it too late to panic? ... EUV is real!" Bob Socha and Frank Abboud collected a distinguished group of invited speakers, who presented their personal knowledge and viewpoint on specific EUV topics, and in a concluding panel discussion gave their assessment of the achievements and challenges with regard to EUV masks.

One additional highlight of the conference surely has been the keynote presentation by Walden C. Rhines, Chairman and Chief Executive Officer of Mentor Graphics, "Bucking the trend: driving changes in how EDA and the semiconductor industries work together," which provided deep insight into long term trends of the semiconductor industry from the viewpoint of EDA. In contrast to photomasks, EDA was up to now able to keep their $2 \%$ share of the semiconductor industry. Mask making may take a lesson or two from EDA's score book!

We thank all of the authors for providing and discussing their insights. We also thank all the members of the program committee for their hard work in helping to make this year's conference a success through their efforts, ranging from reviewing abstracts through chairing sessions. Our sponsors also deserve special thanks for their continued support of Photomask Technology. The SPIE 
staff has our gratitude for their tireless efforts in organizing the conference and ensuring that things ran smoothly as well as their efforts to provide for a timely publication of these proceedings.

We hope you find the papers contained in this proceedings informative and helpful in your professional endeavors.

Wilhelm Maurer

Frank E. Abboud

\section{P.S.}

If you are searching for one of the presentations in Monday's Photomask Japan (PMJ) Session or for one of the posters from PMJ, these are already published in the SPIE Proceedings 7748. 
Thursday Special Session Schedule

\section{Is it too late to PANIC? EUV is real*}

Session Chairs and Panel Moderators: Frank E. Abboud, Intel Corporation (United

States); Robert J. Socha, ASML US, Inc. (United States)

Theme and purpose: EUV is real

"Almost everything in mask making will likely change with EUV: materials, blank inspection, writer compensation, etch processes, metrology, inspection, repair, cleaning, and material handling."

"Opportunities for new innovations and businesses can possibly be the catalyst to push our industry into a new and vibrant industry."

"We need to lead the way and see how we can capitalize on this opportunity."

"We need to get off the short list!"

"Our panel intends to bring this topic to an open and vibrant discussion to get to the real issues for implementation and to weigh pros and cons."

Panelists:

William H. Arnold, ASML US, Inc. (United States)

Vivek K. Singh, Intel Corporation (United States)

Hiroaki Morimoto, Toppan Printing Company, Ltd. (Japan)

Sheng-Ji (Angus) Chin, Taiwan Semiconductor Manufacturing Company Ltd. (Taiwan)

Byung-Gook Kim, SAMSUNG Electronics Company, Ltd. (Korea, Republic of)

Ram Peltinov, Applied Materials (Israel)

Brian L. Haas, KLA-Tencor Corporation (United States)

Oliver O. Kienzle, Carl Zeiss SMS GmbH (Germany)

Yoshiaki Ikuta, Asahi Glass (Japan)

Open Discussion

* For information about the BACUS technical group go to: www.SPIE.org/BACUSHome 
Downloaded From: https://www.spiedigitallibrary.org/conference-proceedings-of-spie on 25 Apr 2023

Terms of Use: https://www.spiedigitallibrary.org/terms-of-use 